\title{
EVALUACIÓN DEL IMPACTO DE LA IMPLEMENTACIÓN DEL PROYECTO PAZ AL AULA EJECUTADO EN EL COLEGIO SAN NICOLÁS DE LA ROCA DE LA CIUDAD DE CARTAGENA
}

\section{Impact assessment of the implementation of peace project executed in the classroom school st nicholas rock city Cartagena}

\author{
Autores: Liliana Camargo Castellar ${ }^{1}$, \\ Asistencia: Nila Hijuelo, Cindy Cortez \\ Correspondencia: liliana.camargo@curnvirtual.edu.co
}

\begin{abstract}
RESUMEN
El presente artículo de investigación realizado desde la perspectiva evaluativa, con enfoque mixto, tiene como objetivo establecer el impacto de la implementación del proyecto en el Colegio San Nicolás de Roca de la ciudad de Cartagena en el año 2012, utilizando para ello el análisis de las encuestas aplicadas a los estudiantes y a los docente y directivas del plantel educativo, complementado con la revisión teórica de la figura de la mediación y el fenómeno social de la violencia escolar.
\end{abstract}

A través de esta investigación se pudo evidenciar que es una preocupación constante de todas las instancias de la sociedad la problemática que se vive actualmente en las instituciones de educación primaria y secundaria en relación con los conflictos que afectan la convivencia escolar, por esta razón todas están comprometidas en combatirla, la Corporación Universitaria Rafael Núñez, viene desarrollando en las instituciones de educación de la ciudad su proyecto PAZ AL AULA, que tiene como eje la educación para la convivencia y la mediación escolar como mecanismo para solucionar el conflicto escolar.

\footnotetext{
${ }^{1}$ Docente investigadora de la facultad de ciencias sociales y humanas, programa derecho de la corporación universitaria Rafael Núñez, Cartagena, especialista en derecho comercial, Magster en desarrollo social.
} 
El análisis de los resultados permitió concluir que luego de la ejecución del proyecto se evidenciaron algunos cambios al interior de la institución educativa, tales como mejoramiento en el clima escolar, a partir de la consideración del dialogo como estrategia fundamental para el abordaje de los conflictos.

\section{Palabras claves:}

Mediación, Conflicto Escolar, Violencia Escolar, Convivencia

\section{ABSTRACT}

This study from evaluation research, with mixed approach aims to establish the impact of project implementation in the San Nicolas de Roca College of Cartagena in 2012, using analysis of surveys applied students and faculty and campus directives, complemented with theoretical revision of the figure of mediation and social phenomenon of school violence.

With this research we could evidence It is a constant concern of all levels of society the problems that currently live in institutions of primary and secondary education in relation to the conflicts affecting school life for this reason all are engaged in combat, the University Corporacion Universitaria Rafael Nuñez it has been developed in educational institutions of the city your project PAZ AL AULA, which is centered education for coexistence and school mediation as a mechanism to solve the school conflict.

The analysis of the results allowed us to conclude that after project implementation some changes within the school, such as improved school climate, from the consideration of dialogue as a key strategy for addressing conflicts were evident.

\section{Keywords:}

Mediation, Conflict School, Coexistence, School violence

\section{INTRODUCCIÓN}

El conflicto escolar es un tema que ha tenido gran trascendencia en el país, el cual enmarca fenómenos muy conocidos para el ambiente escolar como el Bullying o Matoneo, según Enrique Chaux, los estudios indican que en Colombia 1 de cada 5 estudiantes son víctimas del matoneo en todas sus formas..."Colombia es uno", (2012). 
Por lo anterior el Programa de Derecho de Corporación Universitaria Rafael Núñez diseñó el Proyecto Mediación Escolar, el que después de varias experiencias tomo el nombre de Paz al Aula. Este proyecto de intervención se empezó a ejecutar desde el año 2007 en diversas escuelas de la ciudad de Cartagena, en el marco del Proyecto Institucional Escuela Saludable, el cual ha contado con el apoyo de entidades como Telefónica Telecom y Plan Internacional; con este proyecto el programa de derecho dio respuesta a una necesidad comunitaria que luego fue tenida en cuenta por el gobierno nacional quien expidió la Ley 1620 del 15 de marzo del 2013 conocida como la Ley de Convivencia Escolar.

En el año 2012 el proyecto se ejecuta en el Colegio San Nicolás de la Roca, ubicado en el barrio el Prado, Cra. 36 No. 36-61, culminando el mismo año con la puesta en marcha de mesas de mediación. Habiendo transcurrido un año desde su finalización, se da inicio al estudio para establecer el impacto de la implementación de la Mediación Escolar como mecanismo para solucionar el conflicto escolar en el Colegio San Nicolás de la Roca de la Ciudad de Cartagena, para esto se fijan tres objetivos específicos a saber; (I) describir los cambios relevantes relacionados con los factores escolares que afecta la convivencia escolar, a partir de un análisis comparativo de los mismos antes-después y con-sin la ejecución del programa. (II). Analizar si las acciones ejecutadas en el marco del proyecto, generaron cambios en la institución san Nicolás de la Roca. (III). Sistematizar la percepción sobre el impacto social derivado de la aplicación del proyecto paz y aula

Desde el enfoque mixto se realizara la interpretación y análisis de los resultados fundamentada en la investigación evaluativa, cuyo propósito es determinar el éxito o fracaso de la intervención realizada (Babbie, 2000) 
Al finalizar el presente estudio, se pretende obtener un análisis completo de la mediación como herramienta idónea para el abordaje del conflicto escolar y socializar con la institución de educación los resultados obtenidos.

\section{MÉTODOS}

\section{Tipo de estudio}

Esta investigación se realizó desde un enfoque mixto, que combina el método cuantitativo con el cualitativo, con el fin combinar y complementar la información. El método cualitativo es pertinente teniendo en cuenta que a partir de la información recolectada se interpretó de manera general la realidad del clima escolar de la institución educativa. En este sentido Hernández, Fernández y Baptista(2006) afirman que "la investigación cualitativa se fundamenta en una perspectiva interpretativa centrada en el entendimiento del significado de las acciones de los seres vivos, principalmente en los humanos y sus instituciones" (p.9); desde este enfoque la investigación será de tipo interpretativo; por otra parte el método cuantitativo ofrece la posibilidad de analizar el fenómeno a partir de la organización estadística de los datos recogidos, desde este enfoque la investigación será de tipo descriptivo, porque se encargara de describir la situación de la institución educativa después de ejecutado el proyecto, en este sentido Hernández (2000), sostiene que "Los estudios descriptivos únicamente pretenden medir o recoger información de manera independiente o conjunta sobre los conceptos o las variables a las que se refieren..."(p.102)

Por la naturaleza de la misma es una investigación evaluativa, pues tiene el propósito de evaluar el efecto de la intervención social realizada en el año 2012 (Babbie,2000).

\section{Población y muestra}


La población para el presente estudio estuvo constituida por el colegio San Nicolás de la Roca de la ciudad de Cartagena, compuesta por 178 estudiantes, 8 docentes y 2 directivos.

Para la aplicación de la encuesta para establecer el estado actual del clima escolar; la muestra estuvo representada por un grupo alumnos pertenecientes a los cuatro cursos que agrupa a los estudiantes de mayor edad de la institución, el criterio de selección fue la comprensión y no la representatividad estadística; se les aplica a 100 alumnos de los 185 que se encuentran matriculados, cuyas edades oscilan entre los 8 y los 10 años de edad.

En cuanto a la entrevista semi-estructurada, esta se realizó a la totalidad de los docentes y directivos de la institución educativa.

\section{Instrumentos}

Para la recolección de la información necesaria para alcanzar los objetivos de la investigación, se utilizó la encuesta utilizando para ello una adaptación del cuestionario para alumnado de primaria sobre clima escolar y violencia (Ortega y Del Rey, 2001), también se utilizó la entrevistas semi- estructurada a los docentes y directivos del plantel educativo, formulada a partir de una guía administrada por los investigadores, contentiva de preguntas estructurales, de contraste según la clasificación propuesta por Grinnell, y opinión según Mertens, como se citó en Hernández et al. (2006)

\section{Procedimiento}

Encuesta a estudiantes:

Para la aplicación de la encuesta, se adaptó el cuestionario para alumnado de primaria sobre clima escolar y violencia, previo a su aplicación, se realizó una prueba piloto a un grupo de 10 estudiantes conformado por alumnos perteneciente a los diferentes cursos donde se aplicaría el instrumento, se tomó nota de las observaciones y se realizaron 
mínimos ajustes, se acordó la aplicación a todos los cursos y estudiantes que componen la muestra de manera simultánea durante la hora posterior al descanso y por lapso de 45 minutos, con el acompañamiento del docente coordinador del grupo y encuestador.

Entrevista semi estructurada: Para la entrevista semi estructurada, se preparó la guía con preguntas abiertas se entrenó al grupo de gestores sociales facilitadores del proyecto en comunicación, técnicas de entrevista y manejo de emociones, quienes realizaron posteriormente las entrevistas bajo la supervisión del investigador principal, con un tiempo de duración de una hora aproximadamente por entrevistado, en horarios diferentes previamente concertado.

\section{Técnica de análisis y procesamiento de la información}

Una vez recolectada la información se diseñó una matriz en la que se incluyeron 4 categorías de análisis de primer nivel y las categorías de segundo nivel que afloraron en el estudio, para el análisis de la misma se organizaron los datos estadísticamente utilizando para ello el sistema excell, posteriormente se realizó el análisis cualitativo mediante el análisis interpretativo de los datos.

\section{RESULTADOS Y DISCUSIÓN}


Vol.7, No 1. Diciembre de 2015 pp. $94-111$

A continuación se presentan los resultados de la investigación, a partir del análisis de la información obtenida de las dos fuentes utilizadas la entrevista semi- estructurada a docentes y directivos y la encuesta a estudiantes.

Análisis cuantitativo de la encuesta a estudiantes, teniendo en cuenta los dos momentos que involucra el estudio; el anterior a la ejecución del proyecto obtenidos durante el levantamiento de la línea de base y el posterior con los información obtenida de la evaluación del impacto.

\section{Tabla 1:}

Evaluación del Clima escolar en el colegio San Nicolás de Roca

\begin{tabular}{|l|l|l|l|}
\hline $\begin{array}{c}\text { CATEGORÍA } \\
\text { primer nivel }\end{array}$ & $\begin{array}{l}\text { CATEGORIA } \\
\text { Segundo Nivel }\end{array}$ & \multicolumn{1}{|c|}{ LB } & \multicolumn{1}{c|}{ EI } \\
\hline Clima escolar & $\begin{array}{l}\text { Relaciones } \\
\text { sociales }\end{array}$ & $\begin{array}{l}\text { El 63,43\% de la } \\
\text { población se siente } \\
\text { conforme en el } \\
\text { ambiente escolar, en } \\
\text { cambio el 12,74\% es } \\
\text { neutral, y el 6,37\% de } \\
\text { población se siente } \\
\text { conforme en el } \\
\text { ambiente escolar, en } \\
\text { cambio el 20,00\% es } \\
\text { neutral, y el 10,00\% } \\
\text { sienten de forma } \\
\text { regular en la institución }\end{array}$ & $\begin{array}{l}\text { los estudiantes se } \\
\text { sienten de forma } \\
\text { regular en la } \\
\text { institución }\end{array}$ \\
& & & \\
\hline
\end{tabular}

TABLA 2:

Evaluación de la Convivencia escolar en el colegio San Nicolás de la Roca

\begin{tabular}{|l|l|l|l|} 
CATEGORÍA & CATEGORÍA & LB & EI \\
\hline
\end{tabular}


Vol.7, No 1. Diciembre de 2015 pp. $94-111$

\begin{tabular}{|c|c|c|c|}
\hline Primer Nivel & Segundo Nivel & & \\
\hline \multirow[t]{3}{*}{$\begin{array}{l}\text { Convivencia } \\
\text { escolar }\end{array}$} & Entre estudiante & $\begin{array}{l}\text { Un } 46,50 \% \text { de la } \\
\text { población manifiesta } \\
\text { que se ha sentido } \\
\text { agredido por sus } \\
\text { compañeros, mientras }\end{array}$ & $\begin{array}{l}\text { Un } 44,56 \% \text { de la } \\
\text { población manifiesta } \\
\text { que se ha sentido } \\
\text { agredido por sus } \\
\text { compañeros, }\end{array}$ \\
\hline & $\begin{array}{l}\text { Entre estudiante } \\
\text { y docentes }\end{array}$ & $\begin{array}{l}\text { El 58,24\% de la } \\
\text { población manifestó } \\
\text { que nunca ha sido } \\
\text { objeto de violencia por } \\
\text { parte de un profesor o } \\
\text { docente, mientras que } \\
\text { el 10,87\% respondió } \\
\text { que algunas veces, y } \\
\text { el 3,64\% manifestó } \\
\text { que muchas veces }\end{array}$ & $\begin{array}{l}\text { El } 70,85 \% \text { de la } \\
\text { población manifestó } \\
\text { que nunca ha sido } \\
\text { objeto de violencia de } \\
\text { un profesor o docente, } \\
\text { mientras que el } \\
15,80 \% \text { respondió que } \\
\text { algunas veces, y el } \\
\text { 1,78\% manifestó que } \\
\text { muchas veces }\end{array}$ \\
\hline & Otra & $\begin{array}{l}\text { El 51,87\% de la } \\
\text { población manifestó } \\
\text { que nunca ha sido } \\
\text { objeto de violencia por } \\
\text { parte de un adulto, el } \\
9,10 \% \text { manifestó que } \\
\text { algunas veces y el } \\
28,84 \% \text { afirmo que } \\
\text { muchas veces }\end{array}$ & $\begin{array}{l}\text { El 55,80\% de la } \\
\text { población manifestó } \\
\text { que nunca ha sido } \\
\text { objeto de violencia por } \\
\text { parte de un adulto, el } \\
5,60 \% \text { manifestó que } \\
\text { algunas veces y el } \\
30,60 \% \text { afirmo que } \\
\text { muchas veces }\end{array}$ \\
\hline
\end{tabular}


Tabla 3.

Evaluación de la violencia escolar en el Colegio San Nicolás de la Roca

\begin{tabular}{|c|c|c|c|}
\hline $\begin{array}{c}\text { CATEGORÍA } \\
\text { Primer Nivel }\end{array}$ & $\begin{array}{l}\text { CATEGORÍA } \\
\text { Segundo Nivel }\end{array}$ & LB & EI \\
\hline $\begin{array}{l}\text { Violencia } \\
\text { escolar }\end{array}$ & Clase & $\begin{array}{l}\text { Maltrato Verbal: El } \\
44,59 \% \text { manifiesta } \\
\text { que han niños que } \\
\text { maltratan a sus } \\
\text { compañeros, el } \\
21,84 \% \text { niega esta } \\
\text { situación y el 16,38\% } \\
\text { se muestran neutros } \\
\text { frente a la pregunta. } \\
\text { maltrato físico : Un } \\
50,05 \% \text { de la } \\
\text { población declara que } \\
\text { si hay niños violentos } \\
\text { en su grupo, el } 22,75 \% \\
\text { manifiesta que no y el } \\
10,01 \% \text { se muestran }\end{array}$ & $\begin{array}{l}\text { Maltrato Verbal: El } \\
50,75 \% \text { manifiesta } \\
\text { que hay niños que } \\
\text { maltratan a sus } \\
\text { compañeros, el } \\
18,34 \% \text { niega esta } \\
\text { situación y el 9,87\% } \\
\text { se muestran neutros } \\
\text { frente a la pregunta } \\
\text { Maltrato físico: el } \\
39,76 \% \text { de la } \\
\text { población declara que } \\
\text { si hay niños violentos } \\
\text { en su grupo, el } \\
19,65 \% \text { manifiesta } \\
\text { que no y el } 4,70 \% \text { se } \\
\text { muestran neutro }\end{array}$ \\
\hline
\end{tabular}


Vol.7, No 1. Diciembre de 2015 pp. 94 -111

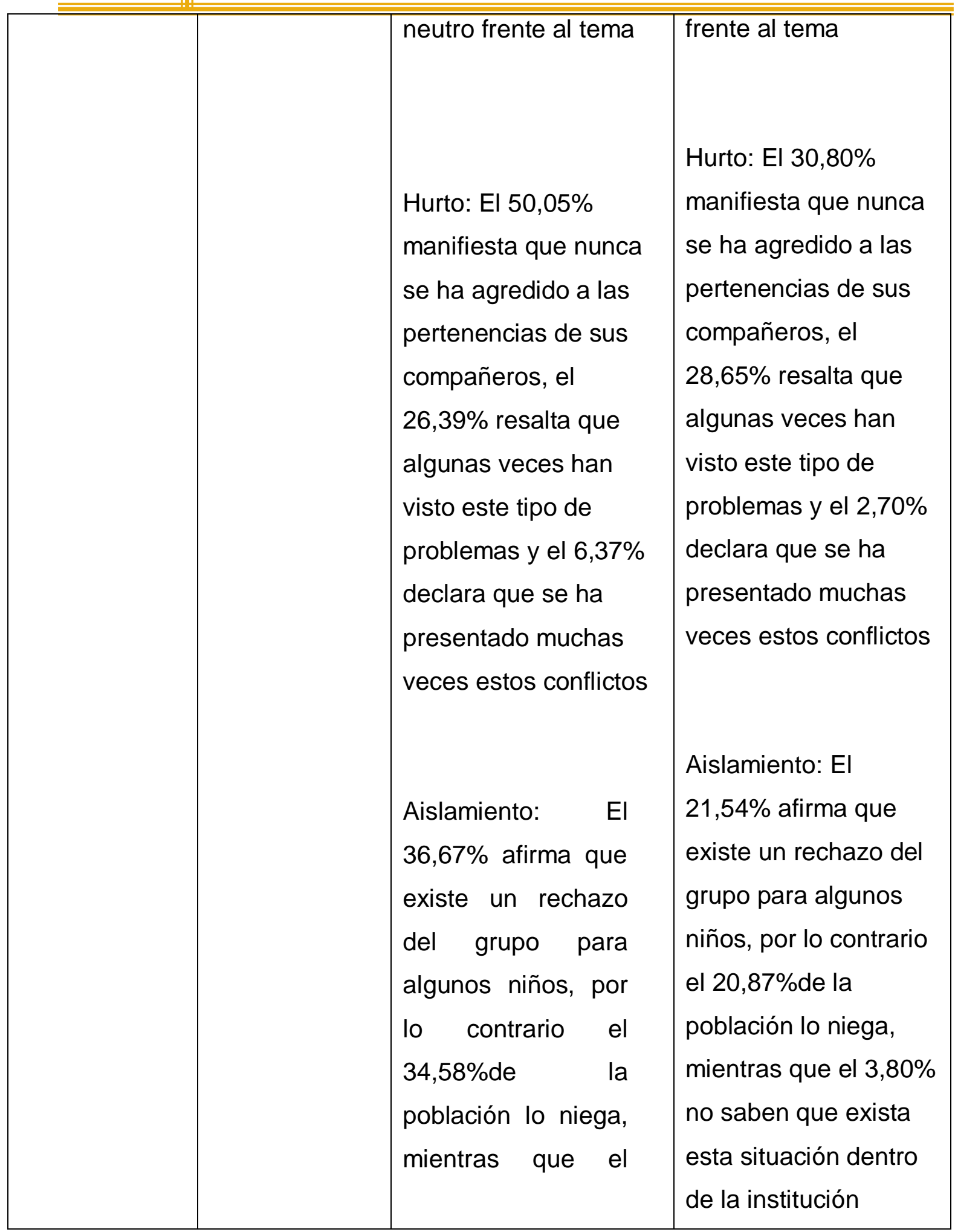


Vol.7, No 1. Diciembre de 2015 pp. $94-111$

\begin{tabular}{|l|l|l|l|}
\hline Intensidad & $\begin{array}{l}13,65 \% \text { no saben } \\
\text { que exista esta } \\
\text { situación dentro de } \\
\text { la institución }\end{array}$ & $\begin{array}{l}\text { El } 69,76 \% \text { de la } \\
\text { población respondió } \\
\text { que nunca ha sido } \\
\text { objeto de violencia por } \\
\text { parte de sus } \\
\text { compañeros, el } \\
15,50 \% \text { manifiesta } \\
\text { que una algunas } \\
\text { veces se ha sentido } \\
\text { violentado por un } \\
\text { compañero y el } 2,00 \%\end{array}$ \\
\hline
\end{tabular}

\section{Tabla 4.}

Evaluación de los mecanismos de abordaje del conflicto en el colegio San Nicolás de la Roca

\begin{tabular}{|c|c|c|c|}
\hline $\begin{array}{c}\text { CATEGORÍA } \\
\text { Primer Nivel }\end{array}$ & $\begin{array}{l}\text { CATEGORÍA } \\
\text { Segundo Nivel }\end{array}$ & $\mathbf{L B}$ & $\mathbf{E I}$ \\
\hline $\begin{array}{l}\text { Tratamiento del } \\
\text { conflicto escolar }\end{array}$ & Mecanismo utilizado & 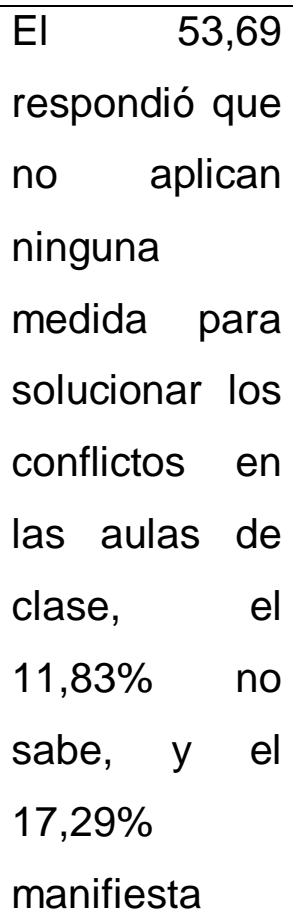 & $\begin{array}{l}\text { El 10,30\% } \\
\text { respondió que } \\
\text { no aplican } \\
\text { ninguna } \\
\text { medida para } \\
\text { solucionar los } \\
\text { conflictos en } \\
\text { las aulas de } \\
\text { clase, el 8,20\% } \\
\text { no sabe, y el } \\
75,87 \% \\
\text { manifiesta que } \\
\text { aplican }\end{array}$ \\
\hline
\end{tabular}


Vol.7, No 1. Diciembre de 2015 pp. $94-111$

\begin{tabular}{|l|l|l|l|}
\hline \hline & & que otro. & $\begin{array}{l}\text { mediación } \\
\text { como solución } \\
\text { de conflictos. }\end{array}$ \\
\hline
\end{tabular}

Análisis cualitativo de la información obtenida a partir de la entrevista a docentes y administrativos y la posterior la triangulación de los métodos.

Clima escolar: El ambiente en el Colegio San Nicolás de la Roca, siempre ha sido bueno, esto no quiere decir que no surjan problemas dentro del aula de clase, la población estudiantil están en edades que hacen a veces difícil la compresión de las situaciones, sin embargo actualmente con la introducción del dialogo y la mediación escolar de conflictos en la cotidianidad de las relaciones, los escolares han comenzado a defenderla y aplicarla como instrumento idóneo para el manejo.

Para la directiva y los docentes antes de la ejecución del proyecto, el colegio se limitaba a utilizar las sanciones consagradas en su manual de convivencia, tales como amonestaciones privadas y expulsión, que por su carácter represivo infunden temor al estudiante. Luego de la intervención los docentes y administrativos enfocan en el dialogo y la mediación escolar la solución de los conflictos, percibiéndose entre los estudiantes un cambio de actitud, que ha repercutido en el mejoramiento del clima escolar haciendo evidente el cambio entre el estado inicial y el posterior a la ejecución del proyecto, sin dejar atrás las reglas de convivencia del colegio la introducción de la mediación escolar ha disminuido los casos de violencia a pesar que aún se presentan conflictos, manifiestan los docentes que estos se solucionan desde un enfoque positivo, debido a que los estudiantes han aprendido a dialogar y solucionar los conflictos utilizando las herramientas adecuadas con el fin de dar por terminado el problema Convivencia escolar: Las relaciones que más afectadas por el conflicto escolar son las que se suscitan entre estudiante, el porcentaje de agresión es alto aunque después de la ejecución del proyecto ha disminuido, al mismo tiempo que revela el estudio que las relaciones entre alumnos y docentes también se ve afectada por el conflicto, pero en 
menor proporción y evidencia una notable mejoría después de la aplicación del proyecto.

Violencia escolar: las manifestaciones de violencia escolar que identifican los estudiantes son el maltrato verbal consistente en insultos, maltrato físico, el hurto conducta que asocian con el uso indebido de las cosas sin permiso o autorización de su dueño, así miso identifican el aislamiento como otra forma de violencia.

Tratamiento del conflicto escolar: En la etapa inicial los estudiantes no conocían otra forma de solucionar los conflictos, la violencia es identificada por la mayoría como la forma eficaz de solucionar de manera inmediata el conflicto, pero a partir d ela ejecuion del proyecto el estudiante ha comenzado a utilizar y recomendar la mediación como otra forma de solucionar los conflictos, actualmente es utilizada dentro de las aulas de clase para mejorar la convivencia escolar.

Los docentes manifiestan que la mediación además de favorecer el clima escolar, aporta a la formación del estudiante, algunos destacaron:

- La medición ha permitido que los estudiantes se involucren en la solución de los conflicto, sin ser parte activa del mismo, buscan contribuir para con el único propósito de mejorar el ambiente escolar.

- El uso de este mecanismos brinda la oportunidad a los estudiantes para que desarrollen sus habilidades de liderazgo, iniciativa, sentido de pertenencia y sobre todo capacidad para controlar las situaciones conflictivas dentro y fuera de las aulas de clases

\section{Discusión:}

Las acciones ejecutadas durante el desarrollo del proyecto Paz al Aula fueron muy beneficiosas para el Colegio San Nicolás de la Roca.

En un primer momento se realizó una sensibilización general a todo el colegio, ya que según Velasco (2013), "lo primero y más importante es crear conciencia del problema y tratar de que no siga ocurriendo" (p.87), es importante que el estudiante conozca sobre el tema y sensibilice frente al mismo. Seguidamente se dio inicio a las 
capacitaciones sobre manejo de conflicto a los grupos seleccionados, es igualmente importante enseñar a los niños las diferente formas que existen para la resolución de los problemas," lo segundo es enseñar a los niños a resolver sus conflictos y buscar diferentes alternativas" (p.87). Por último y no menos importante se capacitó al grupo de estudiantes legitimados por el resto del grupo para actuar como mediadores en cada una de las situaciones conflictivas que se presentaran.

Una vez concluida la primera etapa de sensibilización y capacitación se procedió a La implementación de la herramienta de la Mediación Escolar como alternativa principal para los estudiantes darle solución a sus conflictos, mediante la instalación de la mesas de mediación.

Con la creación de Mesas de Mediación Escolar se crea un nuevo capítulo en la Institución, ya que se abre un espacio al dialogo entre los estudiantes para que ellos logren culturalizarse con esta figura jurídica y resuelvan de manera pacífica y sin la necesidad de optar por la violencia sus diferencias.

Todo lo anterior, sin duda alguna cambió el curso normal del proceso a seguir dentro del Plantel Educativo consagrado en el Manual de Convivencia, el cual de acuerdo a lo tradicional, estaba limitado a la imposición de sanciones al estudiante cuando incurría en conductas de indisciplina que atentaban contra la paz y la tranquilidad de la población estudiantil y por ende perturbaban la convivencia escolar, según González (1994) "los reglamentos escolares generalmente se han pensado y construido como una serie de responsabilidades y deberes de los estudiantes y cuya función es mantener la disciplina del establecimiento educativo y dar trámite a cualquier alteración de la misma" (P.46), constituyéndose en uno de los principales impactos que se destacan de la implementación del proyecto.

La mediación, es el mecanismo idóneo para la resolución de los conflictos escolares, pues esta se adapta a las condiciones y naturaleza del conflicto escolar, en este sentido Núñez, Revelo, Zuluaga (2008) afirman: "la mediación es una modalidad de solución de controversias definidas en forma diferente por las legislaciones nacionales, así como por las supranacionales, de acuerdo con su aplicación y con las facultades 
del tercer mediador, quien está situado y permanece fuera del conflicto para ayudar a las partes a construir el acuerdo." (P.2), por esta razón muchos de los programas propuestos para el abordaje del conflicto escolar tienen como eje central la mediación.

La actitud de los estudiantes frente a esta figura jurídica fue positiva, se ha percibido un cambio en la forma como estos asumen la resolución de sus conflictos, acudiendo a los mediadores escolares capacitados, los actores investigados coinciden en afirmar que la implementación de la figura, lo ha llevado a pensar antes de actuar frente a los estímulos que podrían derivar en una situación conflictiva.

Por otro lado, los docentes al igual que los estudiantes conocieron todo lo relacionado con la mediación como mecanismo alternativo de solución de conflictos, y comprendieron que para la implementación de la misma deben asumir varios compromisos, el primero de ellos consiste en fomentar la comunicación dentro del aula de clase, "es fundamental que en el salón de clase el profesor entable un dialogo para construir puentes de comunicación y así enfrentar problemas, elegir por unanimidad varias opciones o simplemente intercambiar puntos de vista para dar solución en conjunto." (Velasco, 2013, p,93), lo que a su vez supone un cambio en la actitud del docente frente al conflicto, pasando de ser un simple observador que frente al conflicto se encargaba de dinamizar la aplicación de lo establecido en el manual, ratificando su posición de autoridad y poder, a aquel sujeto que interactúa con el estudiante en una relación horizontal donde se reconoce a todos como sujetos de derecho (González, 1994)

Otro compromiso es preparar a los estudiantes de los grados más altos del colegio como mediadores escolares a medida que los otros van egresando de la institución, es entonces el encargado de darle continuidad a la figura de la mediación y de mantener vigente la necesidad del abordaje de los conflictos a partir del diálogo. 


\section{CONCLUSIONES}

El presente estudio realizado para establecer el impacto de la implementación del proyecto Paz al aula en el Colegio San Nicolás de la Roca de la ciudad de Cartagena, a partir de la aplicación de entrevista a los docentes y la directora del colegio y las encuestas aplicadas a los estudiantes, complementado con la revisión teórica sobre el tema permitió comprobar lo siguiente:

La ejecución del proyecto Paz al Aula en el Colegio San Nicolás de la Roca fue exitosa pues la implementación de la herramienta de la Mediación Escolar como mecanismo para que los estudiantes den solución a sus conflictos, impacto positivamente en la escuela, que ha pasado de dar soluciones autoritarias y represivas para transformarse en una escuela donde el conflicto puede dar a aprendizajes significativos, en tal sentido Ramírez, I. (2007) afirma "una buenas institución educativa no es aquella que no tiene conflictos sino la que genera las condiciones adecuadas para abordarlos y que sabe dar respuesta a los mismo, o sea, que favorece el proceso de aprendizaje de sus estudiantes" (p.29) Sin embargo, se hace necesario que este proceso provoque reformas al manual de convivencia.

La actitud de los estudiantes frente a esta figura jurídica fue positiva, teniendo en cuenta que se ha rescatado el valor del respeto y la tolerancia hacia sus compañeros y docentes; se ha percibido un cambio en la forma como asumen la resolución de sus conflictos, acudiendo a los mediadores escolares para restaurar las relaciones con sus compañeros y mantener dentro del plantel educativo una buena convivencia escolar, reconociendo el dialogo como la forma acertada de dar manejo al conflicto.

Los docentes por su parte se comprometieron en fomentar la comunicación para mejorar la relaciones dentro del aula de clase y capacitar como mediadores escolares a los estudiantes de los grados superiores del colegio, así como la enseñanza a los 
Vol.7, No 1. Diciembre de 2015 pp. $94-111$

demás estudiantes acerca de esta nueva herramienta con el fin de erradicar la mínima acción de violencia que se presente dentro y fuera de las aulas de clase.

Actualmente la institución refuerza el Proyecto Paz al Aula, con la inclusión de los temas relacionados con la convivencia en las asignaturas de Democracia y Ética y Valores, por otra parte mensualmente se hace una actividad con todo el personal de la institución, por cada mes escogen un valor para reforzar, a medida que lo realizan logran un espacio para involucrar y unir en un todo la mediación escolar con los valores, además desde la escuela para padre se ha motivado a los mismo para que realicen la misma actividad de mediación en el hogar cuando se presenten problemas con el fin de mejorar y favorecer el ambiente familiar del alumno

\section{REFERENCIAS.}

Babbie, E. (2000). Fundamentos de la Investigación Social. Buenos Aires: Thomson Editores

Colombia es uno de los países con mayores cifras de "matoneo". (16 de marzo de 2012). El Liberal. Recuperado de http://www.mineducacion.gov.co/observatorio/1722/article- 300099.html

Giraldo, J. Los Supuestos Teoricos de la investigacion Socio Juridica. Recuperado de http://www.redsociojuridica.org/documentos/supuestos_teoricos_investigacion_sociojuri dica.pdf

González, M. (1.994) Democracia y escuela. En F. Reyes (Comps). Democracia y conflicto en la escuela. (pp.39-47). Bogotá: Instituto para el desarrollo de la democracia Luis Carlos Galan 
Vol.7, No 1. Diciembre de 2015 pp. $94-111$

Hernández, R., Fernández, C., Baptista, P. (2006). Metodología de la Investigación. (4 ed.). México: Mc Graw Hill

Núñez, J., Revelo, A., Zuluaga, J. (2008). Manual práctico de mediación, El mediador, el procedimiento de la mediación casos de aplicación en propiedad intelectual. Bogotá: Editorial Legis.

Ortega y Del Rey, (2001). Estudio internacional sobre el clima escolar. Cuestionario de primaria. Recuperado de http://convivencia.wordpress.com/2010/05/04/cuestionariopara-alumnado-de-primaria-sobre-clima-escolar-y-violencia/

Ramírez, I. (2007). Equipajes para la convivencia- aprendizaje desde la escuela. En: II laboratorio de paz del oriente antioqueño. Medellín: Instituto popular de capacitación

Velasco, A. (2013) No más bulliyng (1 ed.). Bogotá: Editorial Pianeta 